\title{
Hypermonogenic Functions of Two Vector Variables
}

Eriksson, S. -L.

2018-02

Eriksson, S -L , Orelma , H \& Vieira , N 2018, ' Hypermonogenic Functions of Two Vector Variables ' , Complex Analysis and Operator Theory , vol. 12 , no. 2 , pp. 555-570 . https://doi.org/10.1007/s11785-0

http://hdl.handle.net/10138/307305

https://doi.org/10.1007/s11785-017-0728-7

cc_by_nc_nd

acceptedVersion

Downloaded from Helda, University of Helsinki institutional repository.

This is an electronic reprint of the original article.

This reprint may differ from the original in pagination and typographic detail.

Please cite the original version. 


\title{
Hypermonogenic Functions of Two Vector Variables
}

\author{
S.-L. Eriksson, H. Orelma and N. Vieira
}

\begin{abstract}
In this paper we introduce the modified Dirac operators $\mathcal{M}_{\mathbf{x}}^{\kappa} f:=$ $\partial_{\mathbf{x}} f-\frac{\kappa}{x_{m}} e_{m} \cdot f$ and $\mathcal{M}_{\mathbf{y}}^{\tau} f:=\partial_{\mathbf{y}} f-\frac{\tau}{y_{m}} e_{m} \cdot f$, where $f: \Omega \subset \mathbb{R}_{+}^{m} \times \mathbb{R}_{+}^{m} \rightarrow$ $\mathcal{C} \ell_{0, m}$ is differentiable function, and $\mathcal{C} \ell_{0, m}$ is the Clifford algebra generated by the basis vectors of $\mathbb{R}^{m}$. We look for solutions $f(\mathbf{x}, \mathbf{y})=$ $f\left(\underline{x}, x_{m}, \underline{y}, y_{m}\right)$ of the system $\mathcal{M}_{\mathbf{x}}^{\kappa} f(\mathbf{x}, \mathbf{y})=\mathcal{M}_{\mathbf{y}}^{\tau} f(\mathbf{x}, \mathbf{y})=0$, where the first and third variables are invariant under rotations. These functions are called $(\kappa, \tau)$-hypermonogenic functions. We discuss about axially symmetric functions with respect to the symmetric group $S O(m-1)$. Some examples of axially symmetric $(\kappa, \tau)$-hypermonogenic functions generated by homogeneous functions and hypergeometric functions are presented.
\end{abstract}

Mathematics Subject Classification (2010). Primary 30G35; Secondary 30G20; 30A05.

Keywords. Modified Dirac operator; Axially symmetric functions; Hypermonogenic functions; Several vector variables.

\section{Introduction}

Clifford analysis is nowadays a well know generalization for the classical theory of complex holomorphic function. The theory deals with the functions taking values in a Clifford algebra, which is generated by the elements of the basis of $\mathbb{R}^{m}$ subject to anti-commutative rule. The corresponding function class for complex holomorphic functions is the class of the monogenic functions, which are null solutions of the so-called Dirac or Cauchy-Riemann operators. In Clifford analysis one may construct a function theory with many corresponding properties for the complex case, see $[2,3]$. Classical complex function theory may be extended straightforward to the case of several complex variables assuming holomorphy in each coordinate separately. In the case of Clifford analysis this generalization to several variables is constructed considering Clifford algebra valued functions with several vector variables $($ see $[3,11])$, and we have the system of Dirac operators instead only one. 
Heinz Leutwiler with the first author began to study a modified version of this theory related to hyperbolic spaces (see $[4,6,7]$ ). The main motivation was that the natural generalisation of power functions (which are not monogenic in Clifford's classical analysis) should have some sort of monogeinicity property. They defined the so-called modified Dirac operator with the feature that the power functions also belong to its kernel. The null solutions of this modified Dirac operator are the hypermonogenic functions. Nowadays this "modified Clifford analysis" is designated by hyperbolic function theory, which has also a very complete function theory.

The aim of this paper is to give the first steps in the construction of hyperbolic function theory for functions of two variables. The definition of such theory for the multidimensional case is identical and we leave the systematization of its structure for the future. Here we present the definition of hypermonogenic functions and we show that the set of these functions has non-trivial functions. To show the non-triviality we will study the existence of axially symmetric hypermonogenic functions of two variables by adaptation to the hyperbolic setting of the techniques presented in [11]. We point out that the calculations are more involved in this case.

The classical theory of Dirac operator in Clifford analysis plays an important role in higher dimensional analogy of the theory of holomorphic functions of one complex variables. The essential feature of the Dirac operator is its $S O(m)$ invariance, allowing many theoretical consequences. In the hyperbolic function theory, the fundamental operator (called modified Dirac operator), which is a generalization of the Dirac operator, has weaker symmetry properties. Especially it is symmetric with respect to a subgroup $S O(m-1)$ of $S O(\mathrm{~m})$. The biaxial theory of the Dirac operator is an interesting research topic in current Clifford analysis. The theory plays a big role, for example, in the construction the so-called higher spin Dirac operator (see [14]). In this point of view, it is natural to look also solutions of modified Dirac operator of two vector variables. This paper also works as an example of the systems of Clifford algebra valued operators with more challenging symmetry.

The structure of the paper reads as follows: first we recall some preliminary facts of Clifford and Hyperbolic function theory. In Section 3 we introduce the concept of $(\kappa, \tau)$-hypermonogenic functions and we discuss about axially symmetric functions with respect to the symmetry group $S O(m-1)$. In the last section we present some examples of axially symmetric $(\kappa, \tau)$ hypermonogenic functions generated by some special functions, namely, hypergeometric functions.

\section{Analysis in higher dimension}

In this section we recall some elementary facts about Clifford analysis and hyperbolic theory. 


\subsection{Clifford analysis}

Let $\left\{e_{1}, \ldots, e_{m}\right\}$ be a basis of $\mathbb{R}^{m}$. An arbitrary vector $\mathbf{x} \in \mathbb{R}^{m}$ may be written as a linear combination of the basis vectors as $\mathbf{x}=x_{1} e_{1}+\cdots+x_{m} e_{m}$, where $x_{i} \in \mathbb{R}$ for each $i=1, \ldots, m$. The scalar product of $\mathbf{x}, \mathbf{y} \in \mathbb{R}^{m}$ is defined by $\langle\mathbf{x}, \mathbf{y}\rangle=x_{1} y_{1}+\cdots+x_{n} y_{m}$. The norm for $\mathbf{x} \in \mathbb{R}^{m}$ is defined in terms of the Euclidean scalar product by $|\mathbf{x}|=\sqrt{\mathbf{x} \cdot \mathbf{x}}$. We shall assume that our basis is orthonormal, i.e., $e_{i} \cdot e_{j}=\delta_{i j}$, where $\delta_{i j}$ is the Kronecker symbol. The Clifford product of basis vectors is defined by the anti-commutative rule $e_{i} e_{j}+e_{j} e_{i}=$ $-2 \delta_{i j}$, for each $i, j=1, \ldots, m$. The algebra with respect to these condition is called a Clifford algebra, and denoted by $\mathcal{C} \ell_{0, m}$. The geometric product for any vectors $\mathbf{x}$ and $\mathbf{y}$ may be decomposed into symmetric and antisymmetric parts defined, respectively, by $\mathbf{x} \cdot \mathbf{y}=\frac{1}{2}(\mathbf{x y}+\mathbf{y x})$ and $\mathbf{x} \wedge \mathbf{y}=\frac{1}{2}(\mathbf{x y}-\mathbf{y x})$, where $\mathbf{x}, \mathbf{y} \in \mathbb{R}^{m}$. The antisymmetric part $\mathbf{x} \wedge \mathbf{y}$ is called the outer product and the symmetric part $\mathbf{x} \cdot \mathbf{y}$ is called the inner product. It is easy to prove that the inner product $\mathbf{x} \cdot \mathbf{y}$ is scalar valued and $\mathbf{x} \cdot \mathbf{y}=-\langle\mathbf{x}, \mathbf{y}\rangle$. The inner product may be extended as follows: define the subspaces

$$
\mathcal{C} \ell_{0, m}^{k}=\left\{F_{k}=\sum_{i_{1}<\cdots<i_{k}} F_{i_{1}, \ldots, i_{k}} e_{i_{1}} \cdots e_{i_{k}}: F_{i_{1}, \ldots, i_{k}} \in \mathbb{R}\right\},
$$

then we have the decomposition $\mathcal{C} \ell_{0, m}=\mathbb{R} \oplus \mathbb{R}^{m} \oplus \mathcal{C} \ell_{0, m}^{2} \oplus \ldots \oplus \mathcal{C} \ell_{0, m}^{m}$, where $\mathbb{R}=\mathcal{C} \ell_{0, m}^{0}$ and $\mathcal{C} \ell_{0, m}^{1}=\mathbb{R}^{m}$. Let $[\cdot]_{k}: \mathcal{C} \ell_{0, m} \rightarrow \mathcal{C} \ell_{0, m}^{k}$ be the natural projection. We define $\mathbf{x} \cdot F_{k}=\left[\mathbf{x} F_{k}\right]_{k-1}$ and $\mathbf{x} \cdot F_{0}=0$. Since $f=\sum_{k=0}^{m}[f]_{k}$ we define $\mathbf{x} \cdot f=\sum_{k=0}^{m} \mathbf{x} \cdot[f]_{k}$. Let us consider differentiable functions $f: \Omega \rightarrow \mathcal{C} \ell_{0, m}$, where $\Omega \subset \mathbb{R}^{m}$ is an open subset. In classical Clifford analysis, a fundamental differential operator is the Dirac operator, defined by $\partial_{\mathbf{x}}=\sum_{i=1}^{m} e_{i} \partial_{x_{i}}$. Functions satisfying $\partial_{\mathbf{x}} f=0$ are called left-monogenic functions (resp. rightmonogenic if they satisfy $f \partial_{\mathbf{x}}=0$ ). For more details about Clifford algebras and basic concepts of its associated function theory, we refer the interested reader, for example, to $[2,3]$.

\subsection{Hyperbolic function theory}

A modified approach to the classical Clifford analysis was introduced by Heinz Leutwiler and first author. Their idea was to find an operator such that the power function $\left(x_{0}+\mathbf{x}\right)^{k}$ would be a solution of the operator. Nowadays this approach is called hyperbolic function theory. Our approach here is a slide modification of that theory, since we will working with vector valued operators. A comprehensive introduction to hyperbolic function theory can be found in [5]. The fundamental idea is to work in hyperbolic upper half space, i.e., $\mathbb{R}_{+}^{m}=\left\{\mathbf{x} \in \mathbb{R}^{m}: x_{m}>0\right\}$, which is equipped with the metric $d s^{2}=\frac{d x_{1}^{2}+\cdots+d x_{m}^{2}}{x_{m}^{2}}$. Assume $f: \Omega \rightarrow \mathcal{C} \ell_{0, m}$ is a differentiable function, where $\Omega \subset \mathbb{R}_{+}^{m}$ is an open subset. The so called modified Dirac operator is defined by $\mathcal{M}_{\mathbf{x}}^{\kappa} f:=\partial_{\mathbf{x}} f-\frac{\kappa}{x_{m}} e_{m} \cdot f$, where $\kappa$ is an arbitrary real parameter. If $\mathcal{M}_{\mathbf{x}}^{\kappa} f=$ 0 , the function $f$ is called $\kappa$-hypermonogenic. Note that 0 -hypermonogenic functions correspond to monogenic functions. Other interesting values of $\kappa$ 
are $\kappa=m-2 k$, where $k=0, \ldots, m$, which are related to harmonic differential forms in the upper half space (for more details see [8]).

\section{3. $(\kappa, \tau)$-Hypermonogenic Functions}

The idea of this paper is to study hypermonogenic functions of two vector variables. We will consider differentiable functions $f: \Omega \rightarrow \mathcal{C} \ell_{0, m}$, where $\Omega \subset \mathbb{R}_{+}^{m} \times \mathbb{R}_{+}^{m}$ is an open subset. For a function $f=f(\mathbf{x}, \mathbf{y})$, we define modified Dirac operators

$$
\mathcal{M}_{\mathbf{x}}^{\kappa} f:=\partial_{\mathbf{x}} f-\frac{\kappa}{x_{m}} e_{m} \cdot f \quad \text { and } \quad \mathcal{M}_{\mathbf{y}}^{\tau} f:=\partial_{\mathbf{y}} f-\frac{\tau}{y_{m}} e_{m} \cdot f .
$$

Then we can introduce the following definition.

Definition 3.1. A differentiable function $f: \Omega \subset \mathbb{R}_{+}^{m} \times \mathbb{R}_{+}^{m} \rightarrow \mathcal{C} \ell_{0, m}$ is $(\kappa, \tau)$ hypermonogenic if

$$
\left\{\begin{array}{l}
\mathcal{M}_{\mathbf{x}}^{\kappa} f(\mathbf{x}, \mathbf{y})=0 \\
\mathcal{M}_{\mathbf{y}}^{\tau} f(\mathbf{x}, \mathbf{y})=0
\end{array} .\right.
$$

Note that variables $\mathbf{x}$ and $\mathbf{y}$ live in the same space $\mathbb{R}_{+}^{m}$, and therefore it is possible that $\langle\mathbf{x}, \mathbf{y}\rangle \neq 0$.

\subsection{Axially symmetric functions}

We consider a function of the splitted variables $f: \Omega \subset \mathbb{R}_{+}^{m} \times \mathbb{R}_{+}^{m} \rightarrow \mathcal{C} \ell_{0, m}$, such that $\mathbf{x}=\underline{x}+x_{m} e_{m}$ and $\mathbf{y}=\underline{y}+y_{m} e_{m}$. Then we have functions $f=f\left(\underline{x}, x_{m}, \underline{y}, y_{m}\right)$ and these are $S O(m-1)$-invariant functions w.r.t. $\underline{x}$ and $\underline{y}$. We note that $e_{m} \underline{x}=-\underline{x} e_{m}, e_{m} \underline{y}=-\underline{y} e_{m}$ and $e_{m} \underline{x} \underline{y}=\underline{x} \underline{y} e_{m}$. Moreover, all $S O(m-1)$-invariant functions are essentially linear combinations of the elements of the following set of functions

$$
\left\{A_{1}, \underline{x} A_{2}, \underline{y} A_{3}, \underline{x} \underline{y} A_{4}, e_{m} A_{5}, \underline{x} e_{m} A_{6}, \underline{y} e_{m} A_{7}, \underline{x} \underline{y} e_{m} A_{8}\right\},
$$

where $A_{j}$ are functions of five axially coordinates $|\underline{x}|^{2}, x_{m},|\underline{y}|^{2}, y_{m},\langle\underline{x}, y\rangle$. That kind of functions in Euclidean case are defined in [11-13]. The aim of the Section 4 is to present some concrete examples of axially symmetric $(\kappa, \tau)$-hypermonogenic functions, in order to show that the set of theses functions is non-trivial. Before that we collect some results about the behaviour of the defined modified Dirac operators $\mathcal{M}_{\mathbf{x}}^{\kappa}$ and $\mathcal{M}_{\mathbf{y}}^{\tau}$.

\subsection{Action of operators}

Here, we present some results about the action of operators $\mathcal{M}_{\mathbf{x}}^{\kappa}$ and $\mathcal{M}_{\mathbf{y}}^{\tau}$ over the preceding axially symmetric functions. This first result collects several relations presented and proved in [11]. 
Proposition 3.2. (cf. [11, Sec.4]) For a differentiable real valued function $A=A\left(a, x_{m}, b, y_{m}, c\right)$, with $a=|\underline{x}|^{2}, b=|y|^{2}$ and $c=\langle\underline{x}, y\rangle$, the following relations hold

$$
\begin{aligned}
& \partial_{\underline{x}}(A)=2 \underline{x} \partial_{a} A+\underline{y} \partial_{c} A, \\
& \partial_{\underline{x}}(\underline{x} A)=-\left[(m-1)+2 a \partial_{a}+2 c \partial_{c}\right] A-\underline{x} \underline{y} \partial_{c} A, \\
& \partial_{\underline{x}}(\underline{y} A)=-b \partial_{c} A+2 \underline{x} \underline{y} \partial_{a} A, \\
& \partial_{\underline{x}}(\underline{x} \underline{y} A)=-\left[(m-1)+2 a \partial_{a}+2 c \partial_{c}\right] \underline{y} A+b \underline{x} \partial_{c} A .
\end{aligned}
$$

Using these we have the following result

Proposition 3.3. Let $a=|\underline{x}|^{2}, b=|\underline{y}|^{2}$ and $c=\langle\underline{x}, \underline{y}\rangle$. The action of the operator $\mathcal{M}_{\mathbf{x}}^{\kappa}$ over each element of (3.2) is given by (where we use the short notation $A=A_{j}$, for each $\left.j=1, \ldots, 8\right)$ :

$$
\begin{aligned}
& \mathcal{M}_{\mathbf{x}}^{\kappa}(A)=2 \underline{x} \partial_{a} A+\underline{y} \partial_{c} A+e_{m} \partial_{x_{m}} A, \\
& \mathcal{M}_{\mathbf{x}}^{\kappa}(\underline{x} A)=-\left[(m-1)+2 a \partial_{a}+2 c \partial_{c}\right] A-\underline{x} \underline{y} \partial_{c} A-\underline{x} e_{m} \partial_{x_{m}} A, \\
& \mathcal{M}_{\mathbf{x}}^{\kappa}(\underline{y} A)=-b \partial_{c} A+2 \underline{x} \underline{y} \partial_{a} A-\underline{y} e_{m} \partial_{x_{m}} A, \\
& \mathcal{M}_{\mathbf{x}}^{\kappa}(\underline{x} \underline{y} A)=b \underline{x} \partial_{c} A-\left[(m-1)+2 a \partial_{a}+2 c \partial_{c}\right] \underline{y} A+\underline{x} \underline{y} e_{m} \partial_{x_{m}} A, \\
& \mathcal{M}_{\mathbf{x}}^{\kappa}\left(e_{m} A\right)=-\left(\partial_{x_{m}}-\frac{\kappa}{x_{m}}\right) A+2 \underline{x} e_{m} \partial_{a} A+\underline{y} e_{m} \partial_{c} A, \\
& \mathcal{M}_{\mathbf{x}}^{\kappa}\left(\underline{x} e_{m} A\right)=\left(\partial_{x_{m}}-\frac{\kappa}{x_{m}}\right) \underline{x} A-\left[(m-1)+2 a \partial_{a}+2 c \partial_{c}\right] e_{m} A \\
& \quad-\underline{x} e_{m} \partial_{c} A, \\
& \mathcal{M}_{\mathbf{x}}^{\kappa}\left(\underline{y} e_{m} A\right)=\left(\partial_{x_{m}}-\frac{\kappa}{x_{m}}\right) \underline{y} A-b e_{m} \partial_{c} A+2 \underline{x} \underline{y} e_{m} \partial_{a} A, \\
& \mathcal{M}_{\mathbf{x}}^{\kappa}\left(\underline{x} \underline{y} e_{m} A\right)=-\left(\partial_{x_{m}}-\frac{\kappa}{x_{m}}\right) \underline{x} \underline{y} A+b \underline{x} e_{m} \partial_{c} A \\
& \quad-\left[(m-1)+2 a \partial_{a}+2 c \partial_{c}\right] \underline{y} e_{m} A .
\end{aligned}
$$

The proof of this relations comes from straightforward calculations. However, we point out that if a function $f$ does not have the $e_{m}$-component, i.e., $e_{m} \cdot f=0$, we have $\mathcal{M}_{\mathbf{x}}^{\kappa}\left(e_{m} f\right)=-\partial_{x_{m}} f+\frac{\kappa}{x_{m}} f-e_{m} \partial_{\underline{x}} f$. This remark allow us to deduce more directly the last four relations.

A similar result can be obtained for the operator $\mathcal{M}_{\mathbf{y}}^{\tau} f=\partial_{\mathbf{y}} f-\frac{\tau}{y_{m}} e_{m} \cdot f$. The easiest way to derive it, is to change the roles of $\underline{x}$ to $\underline{y}, x_{m}$ to $y_{m}, a$ to $b, \kappa$ to $\tau$, and use the relation $\underline{y} \underline{x}=-2 c-\underline{x} \underline{y}$. Hence we get 
Proposition 3.4. Let $a=|\underline{x}|^{2}, b=|\underline{y}|^{2}$ and $c=\langle\underline{x}, \underline{y}\rangle$. The action of the operator $\mathcal{M}_{\mathbf{y}}^{\tau}$ over each element of (3.2) is given by (where we use the short notation $A=A_{j}$, for each $\left.j=1, \ldots, 8\right)$ :

$$
\begin{aligned}
& \mathcal{M}_{\mathbf{y}}^{\tau}(A)=\underline{x} \partial_{c} A+2 \underline{y} \partial_{b} A+e_{m} \partial_{y_{m}} A, \\
& \mathcal{M}_{\mathbf{y}}^{\tau}(\underline{x} A)=-\left(4 c \partial_{b}+a \partial_{c}\right) A-2 \underline{x} \underline{y} \partial_{b} A-\underline{x} e_{m} \partial_{y_{m}} A, \\
& \mathcal{M}_{\mathbf{y}}^{\tau}(\underline{y} A)=-\left[(m-1)+2 b \partial_{b}\right] A+\underline{x} \underline{y} \partial_{c} A-\underline{y} e_{m} \partial_{y_{m}} A, \\
& \mathcal{M}_{\mathbf{y}}^{\tau}(\underline{x} \underline{y} A)=\left[(m-3)-2 b \partial_{b}\right] \underline{x} A-\left(4 c \partial_{b}+a \partial_{c}\right) \underline{y} A+\underline{x} \underline{y} e_{m} \partial_{y_{m}} A, \\
& \mathcal{M}_{\mathbf{y}}^{\tau}\left(e_{m} A\right)=-\left(\partial_{y_{m}}-\frac{\tau}{y_{m}}\right) A+\underline{x} e_{m} \partial_{c} A+2 \underline{y} e_{m} \partial_{b} A, \\
& \mathcal{M}_{\mathbf{y}}^{\tau}\left(\underline{x} e_{m} A\right)=\left(\partial_{y_{m}}-\frac{\tau}{y_{m}}\right) \underline{x} A-\left(4 c \partial_{b}+a \partial_{c}\right) e_{m} A-2 \underline{x} \underline{y} e_{m} \partial_{b} A, \\
& \mathcal{M}_{\mathbf{y}}^{\tau}\left(\underline{y} e_{m} A\right)=\left(\partial_{y_{m}}-\frac{\tau}{y_{m}}\right) \underline{y} A-\left[(m-1)+2 b \partial_{b}\right] e_{m} A+\underline{x} \underline{y} e_{m} \partial_{c} A, \\
& \mathcal{M}_{\mathbf{y}}^{\tau}\left(\underline{x} \underline{y} e_{m} A\right)=-\left(\partial_{y_{m}}-\frac{\tau}{y_{m}}\right) \underline{x} \underline{y} A+\left[(m-3)-2 b \partial_{b}\right] \underline{x} e_{m} A \\
& \quad-\left(4 c \partial_{b}+a \partial_{c}\right) \underline{y} e_{m} A .
\end{aligned}
$$

Proof. The first three relations come directly. The fourth is not that straightforward than the previous three. In fact, first we compute

$$
\partial_{\underline{y}}(\underline{x} \underline{y})=\sum_{i, j=1}^{m-1} \partial_{y_{i}} y_{j} e_{i} \underline{x} e_{j}=\sum_{j=1}^{m-1} e_{j} \underline{x} e_{j}=(m-3) \underline{x} \text {. }
$$

Then we have

$$
\begin{aligned}
\mathcal{M}_{\mathbf{y}}^{\tau}(\underline{x} \underline{y} A) & =\partial_{\underline{y}} A \underline{x} \underline{y}+\partial_{\underline{y}}(\underline{x} \underline{y}) A+\underline{x} \underline{y} e_{m} \partial_{x_{m}} A \\
& =2 \underline{y} \underline{x} \underline{y} \partial_{b} A-a \underline{y} \partial_{c} A+(m-3) \underline{x} A+\underline{x} \underline{y} e_{m} \partial_{x_{m}} A .
\end{aligned}
$$

From the fact that $\underline{y} \underline{x} \underline{y}=-(b \underline{x}+2 c \underline{y})$, we obtain the fourth relation. To obtain the last four relations we observe (as we had done for $\mathcal{M}_{\mathbf{x}}^{\kappa}$ ) that if a function $f$ does not have the $e_{m}$-component, i.e., $e_{m} \cdot f=0$, we have $\mathcal{M}_{\mathbf{y}}^{\tau}\left(e_{m} f\right)=-\partial_{y_{m}} f+\frac{\tau}{y_{m}} f-e_{m} \partial_{\underline{y}} f$.

The mapping relations of the operators $\mathcal{M}_{\mathbf{x}}^{\kappa}$ and $\mathcal{M}_{\mathbf{y}}^{\tau}$ over axially symmetric functions is resumed in the following table (the table is the same to both operator up to constants): 


\begin{tabular}{|c|c|c|c|c|c|c|c|c|}
\hline \hline $\mathcal{M}_{\mathbf{x}}^{\kappa}, \mathcal{M}_{\mathbf{y}}^{\tau}$ & $A$ & $\underline{x} A$ & $\underline{y} A$ & $\underline{x} \underline{y} A$ & $e_{m} A$ & $\underline{x} e_{m} A$ & $\underline{y} e_{m} A$ & $\underline{x} \underline{y} e_{m} A$ \\
\hline \hline$A$ & & $\bullet$ & $\bullet$ & & $\bullet$ & & & \\
\hline$\underline{x} A$ & $\bullet$ & & & $\bullet$ & & $\bullet$ & & \\
\hline$\underline{y} A$ & $\bullet$ & & & $\bullet$ & & & $\bullet$ & \\
\hline$\underline{x} \underline{y} A$ & & $\bullet$ & $\bullet$ & & & & & $\bullet$ \\
\hline$e_{m} A$ & $\bullet$ & & & & & $\bullet$ & $\bullet$ & \\
\hline$\underline{x} e_{m} A$ & & $\bullet$ & & & $\bullet$ & & & $\bullet$ \\
\hline$\underline{y} e_{m} A$ & & & $\bullet$ & & $\bullet$ & & & $\bullet$ \\
\hline$\underline{x} \underline{y} e_{m} A$ & & & & $\bullet$ & & $\bullet$ & $\bullet$ & \\
\hline \hline
\end{tabular}

\section{Examples of axially symmetric $(\kappa, \tau)$-hypermonogenic functions}

In this section we use the relations presented in Propositions 3.3 and 3.4 to obtain some examples of axially symmetric $(\kappa, \tau)$-hypermonogenic functions. We observe that all the examples presented in this section do not correspond to trivial cases.

4.1. Axial symmetric $(\kappa, \tau)$-hypermonogenic functions of the form $e_{m} A$ Taking into account Propositions 3.3 and 3.4, the system (3.1) takes the form

$$
\left\{\begin{array}{l}
\mathcal{M}_{\mathbf{x}}^{\kappa}\left(e_{m} A\right)=-\partial_{x_{m}} A+\frac{\kappa}{x_{m}} A+2 \underline{x} e_{m} \partial_{a} A+\underline{y} e_{m} \partial_{c} A=0 \\
\mathcal{M}_{\mathbf{y}}^{\tau}\left(e_{m} A\right)=-\partial_{y_{m}} A+\frac{\tau}{y_{m}} A+2 \underline{y} e_{m} \partial_{b} A+\underline{x} e_{m} \partial_{c} A=0 .
\end{array},\right.
$$

which is equivalent to

$$
\left\{\begin{array}{l}
\partial_{x_{m}} A-\frac{\kappa}{x_{m}} A=0 \\
\partial_{y_{m}} A-\frac{\tau}{y_{m}} A=0 \\
\partial_{a} A=\partial_{b} A=\partial_{c} A=0
\end{array} .\right.
$$

Solving the previous system we get immediately that $A=x_{m}^{\kappa} y_{m}^{\tau}$, and therefore we get the following $(\kappa, \tau)$-hypermonogenic function

$$
f(\mathbf{x}, \mathbf{y})=e_{m} x_{m}^{\kappa} y_{m}^{\tau}
$$


4.2. Axial symmetric $(\kappa, \tau)$-hypermonogenic functions of the form $(\underline{x} A+$ $\underline{y} B) e_{m}$

Taking into account Propositions 3.3 and 3.4, the system (3.1) becomes

$$
\left\{\begin{array}{l}
\mathcal{M}_{\mathbf{x}}^{\kappa}\left(\underline{x} e_{m} A+\underline{y} e_{m} B\right) \\
=\underline{x} \partial_{x_{m}} A-\frac{\kappa}{x_{m}} \underline{x} A-(m-1) e_{m} A-2 a e_{m} \partial_{a} A \\
-(\underline{x} \underline{y}+2 c) e_{m} \partial_{c} A+\underline{y} \partial_{x_{m}} B-\frac{\kappa}{x_{m}} \underline{y} B+2 \underline{x} \underline{y} e_{m} \partial_{a} B-b e_{m} \partial_{c} B=0 \\
\mathcal{M}_{\mathbf{y}}^{\tau}\left(\underline{x} e_{m} A+\underline{y} e_{m} B\right) \\
=\underline{x} \partial_{y_{m}} A-\frac{y_{m}}{y_{m}} A-2(\underline{x} \underline{y}+2 c) e_{m} \partial_{b} A-a e_{m} \partial_{c} A \\
+\underline{y} \partial_{y_{m}} B-\frac{y_{m}}{y_{m}} B-(m-1) e_{m} B-2 b e_{m} \partial_{b} B+\underline{x} \underline{y} e_{m} \partial_{c} B=0 .
\end{array}\right.
$$

The previous system is equivalent to

$$
\left\{\begin{array}{l}
\partial_{x_{m}} A-\frac{\kappa}{x_{m}} A=0 \\
2 \partial_{a} B-\partial_{c} A=0 \\
2 a \partial_{a} A+2 c \partial_{c} A+b \partial_{c} B+(m-1) A=0 \\
\partial_{x_{m}} B-\frac{\kappa}{x_{m}} B=0 \\
\partial_{y_{m}} A-\frac{\tau}{y_{m}} A=0 \\
2 \partial_{b} A-\partial_{c} B=0 \\
4 c \partial_{b} A+a \partial_{c} A+2 b \partial_{b} B+(m-1) B=0 \\
\partial_{y_{m}} B-\frac{\tau}{y_{m}} B=0
\end{array}\right.
$$

Taking into account that $A=u(a, b, c) x_{m}^{\kappa} y_{m}^{\tau}$ and $B=v(a, b, c) x_{m}^{\kappa} y_{m}^{\tau}$, then (4.1) reduces to

$$
\left\{\begin{array}{l}
2 \partial_{a} v-\partial_{c} u=0 \\
2 a \partial_{a} u+2 c \partial_{c} u+b \partial_{c} v+(m-1) u=0 \\
2 \partial_{b} u-\partial_{c} v=0 \\
4 c \partial_{b} u+a \partial_{c} u+2 b \partial_{b} v+(m-1) v=0
\end{array} .\right.
$$

If $c=0$, i.e., $\mathbf{x}$ and $\mathbf{y}$ are orthogonal, system (4.2) becomes

$$
\left\{\begin{array}{l}
\partial_{a} v=0 \\
2 a \partial_{a} u+(m-1) u=0 \\
\partial_{b} u=0 \\
2 b \partial_{b} v+(m-1) v=0
\end{array} .\right.
$$


and then

$$
\begin{aligned}
& u(a, b, c)=u(a)=a^{\frac{1-m}{2}} \quad \Rightarrow \quad A=a^{\frac{1-m}{2}} x_{m}^{\kappa} y_{m}^{\tau}, \\
& v(a, b, c)=v(b)=b^{\frac{1-m}{2}} \Rightarrow B=b^{\frac{1-m}{2}} x_{m}^{\kappa} y_{m}^{\tau},
\end{aligned}
$$

where $a=|\underline{x}|^{2}, b=|y|^{2}$. Hence we obtain the following axially symmetric $(\kappa, \tau)$-hypermonogenic function

$$
f(\mathbf{x}, \mathbf{y})=x_{m}^{\kappa} y_{m}^{\tau}\left(\underline{x}|\underline{x}|^{1-m}+\underline{y}|\underline{y}|^{1-m}\right) e_{m} .
$$

On the other hand, if $c \neq 0$, system (4.2) reduces to the following system of two independent partial differential equations

$$
\left\{\begin{array}{l}
a \partial_{a} u+b \partial_{b} u+c \partial_{c} u=\frac{1-m}{2} u \\
a \partial_{a} v+b \partial_{b} v+c \partial_{c} v=\frac{1-m}{2} v
\end{array},\right.
$$

under the condition that $u$ and $v$ are solutions of

$$
\left\{\begin{array}{l}
2 \partial_{a} v-\partial_{c} u=0 \\
2 \partial_{b} u-\partial_{c} v=0
\end{array} .\right.
$$

Taking into account Equation (8.2.1.7) in [10] we conclude that the solution of each equation is given by

$$
u(a, b, c)=c^{\frac{1-m}{2}} \Phi_{1}\left(\frac{a}{c}, \frac{b}{c}\right) \quad v(a, b, c)=c^{\frac{1-m}{2}} \Phi_{2}\left(\frac{a}{c}, \frac{b}{c}\right)
$$

where $\Phi_{1}$ and $\Phi_{2}$ are two arbitrary differentiable functions of two variables. These functions $u$ and $v$ should be solutions of (4.4), i.e., after substituting them in (4.4) they must be solution of the following Vekua-type system

$$
\left\{\begin{array}{c}
t \partial_{t} \Phi_{1}+s \partial_{s} \Phi_{1}+2 \partial_{t} \Phi_{2}-\frac{1-m}{2} \Phi_{1}=0 \\
t \partial_{t} \Phi_{2}+s \partial_{s} \Phi_{2}+2 \partial_{s} \Phi_{1}-\frac{1-m}{2} \Phi_{2}=0
\end{array}\right.
$$

where $t=\frac{a}{c}$ and $s=\frac{b}{c}$. It remains now to obtain some examples of $\Phi_{1}$ and $\Phi_{2}$, which are solutions of (4.6).

4.2.1. Case when $\Phi_{1}$ and $\Phi_{2}$ are homogeneous functions. The easiest possible solution for (4.6) is when $\Phi_{1}$ and $\Phi_{2}$ are $\frac{1-m}{2}$-homogeneous, i.e., $\Phi_{1}$ and $\Phi_{2}$ are of the form

$$
\Phi_{1}(t, s)=\sum_{j=0}^{m-1} \alpha_{j} t^{-\frac{j}{2}} s^{\frac{1-m+j}{2}} \quad \Phi_{2}(t, s)=\sum_{j=0}^{m-1} \beta_{j} t^{-\frac{j}{2}} s^{\frac{1-m+j}{2}},
$$

where $\alpha_{j}$ and $\beta_{j}$ are real coefficients. Substituting $\Phi_{1}$ and $\Phi_{2}$ into (4.6) we obtain

$$
\left\{\begin{array}{l}
\partial_{t} \Phi_{2}=0 \\
\partial_{s} \Phi_{1}=0
\end{array}\right.
$$


Takin in account (4.7) the previous system becomes

$$
\left\{\begin{array}{l}
-\sum_{j=0}^{m-1} \frac{j}{2} \beta_{j} t^{-\frac{j+2}{2}} s^{\frac{1-m+j}{2}}=0 \\
\sum_{j=0}^{m-1} \frac{1-m+j}{2} \alpha_{j} t^{-\frac{j}{2}} s^{\frac{-m+j-1}{2}}=0
\end{array},\right.
$$

and therefore we conclude that the only possible solution are, up to a constant, given by

$$
\Phi_{1}(t, s)=t^{\frac{1-m}{2}} \quad \text { and } \quad \Phi_{2}(t, s)=s^{\frac{1-m}{2}} .
$$

Taking into account that $t=\frac{a}{c}$ and $s=\frac{b}{c}$, we have, from the expressions of $\Phi_{1}$ and $\Phi_{2}$, that

$$
\begin{aligned}
& u(a, b, c)=c^{\frac{1-m}{2}} t^{\frac{1-m}{2}}=a^{\frac{1-m}{2}} \Rightarrow A=a^{\frac{1-m}{2}} x_{m}^{\kappa} y_{m}^{\tau}, \\
& v(a, b, c)=c^{\frac{1-m}{2}} s^{\frac{1-m}{2}}=b^{\frac{1-m}{2}} \Rightarrow B=b^{\frac{1-m}{2}} x_{m}^{\kappa} y_{m}^{\tau},
\end{aligned}
$$

where $a=|\underline{x}|^{2}, b=|\underline{y}|^{2}$. Consequently we obtain the following axially symmetric $(\kappa, \tau)$-hypermonogenic function

$$
f(\mathbf{x}, \mathbf{y})=x_{m}^{\kappa} y_{m}^{\tau}\left(\underline{x}|\underline{x}|^{1-m}+\underline{y}|\underline{y}|^{1-m}\right) e_{m} .
$$

It is imediate that (4.8) coincides with (4.3).

4.2.2. Case when $\Phi_{1}$ and $\Phi_{2}$ are hypergeometric functions. Let us consider that the solutions $\Phi_{1}$ and $\Phi_{2}$ of (4.6) are of the form

$$
\Phi_{1}(t, s)=\sum_{j=0}^{+\infty} t^{j} A_{j}(s) \quad \text { and } \quad \Phi_{2}(t, s)=\sum_{j=0}^{+\infty} t^{j} B_{j}(s) .
$$

Substituting (4.9) into (4.6) we obtain

$$
\left\{\begin{array}{l}
\sum_{j=0}^{+\infty} t^{j}\left[s A_{j}^{\prime}(s)+\frac{m+2 j-1}{2} A_{j}(s)+2(j+1) B_{j+1}(s)\right]=0 \\
\sum_{j=0}^{+\infty} t^{j}\left[s B_{j}^{\prime}(s)+\frac{m+2 j-1}{2} B_{j}(s)+2 A_{j}^{\prime}(s)\right]=0
\end{array}\right.
$$

which leads to

$$
\left\{\begin{array}{l}
s A_{j}^{\prime}(s)+\frac{m+2 j-1}{2} A_{j}(s)+2(j+1) B_{j+1}(s)=0 \\
s B_{j}^{\prime}(s)+\frac{m+2 j-1}{2} B_{j}(s)+2 A_{j}^{\prime}(s)=0
\end{array},\right.
$$

with $j=0,1,2, \ldots$. Taking derivatives with respect to $s$ of both members of the first equality in (4.10), we get

$$
s A_{j}^{\prime \prime}(s)+\frac{m+2 j+1}{2} A_{j}^{\prime}(s)+2(j+1) B_{j+1}^{\prime}(s),
$$


with $j=0,1,2, \ldots$. On the other hand, from the second equation of $(4.10)$ we have the following two relations

$$
\begin{array}{ll}
A_{j}^{\prime}(s)=-\frac{s}{2} B_{j}^{\prime}(s)-\frac{m+2 j-1}{4} B_{j}(s), & j=0,1,2, \ldots \\
A_{j}^{\prime \prime}(s)=-\frac{s}{2} B_{j}^{\prime \prime}(s)-\frac{m+2 j+1}{4} B_{j}^{\prime}(s), \quad j=0,1,2, \ldots
\end{array}
$$

Substituting (4.12) and (4.13) into (4.11) we get

$$
\begin{array}{r}
-\frac{s^{2}}{2} B_{j}^{\prime \prime}(s)-\frac{(m+2 j+1) s}{2} B_{j}^{\prime}(s)-\frac{(m+2 j)^{2}-1}{8} B_{j}(s) \\
+2(j+1) B_{j+1}^{\prime}(s)=0
\end{array}
$$

with $\quad j=0,1,2, \ldots$, that is

$$
\begin{aligned}
& B_{j+1}^{\prime}(s) \\
& \quad=\frac{1}{2(j+1)}\left[\frac{s^{2}}{2} B_{j}^{\prime \prime}(s)+\frac{(m+2 j+1) s}{2} B_{j}^{\prime}(s)+\frac{(m+2 j)^{2}-1}{8} B_{j}(s)\right]
\end{aligned}
$$

with $j=0,1,2, \ldots$ In order to solve (4.15) we start looking for solutions of the differential equation

$$
B_{j}(s)=\frac{s^{2}}{2} B_{j}^{\prime \prime}(s)+\frac{(m+2 j+1) s}{2} B_{j}^{\prime}(s)+\frac{(m+2 j)^{2}-1}{8} B_{j}(s),
$$

with $j=0,1,2, \ldots$, which is equivalent to

$$
s^{2} B_{j}^{\prime \prime}(s)+(m+2 j+1) s B_{j}^{\prime}(s)+\frac{(m+2 j)^{2}-9}{4} B_{j}(s)=0,
$$

with $j=0,1,2, \ldots$ Equation (4.16) corresponds to the so-called Euler's equation and its solution can be found in several handbooks of ordinary differential equations (see, for example, Equation (2.1.2-4.123) with $a=m+$ $2 j+1$ and $b=\frac{(m+2 j)^{2}-9}{4}$ such that $(1-a)^{2}>4 b$ in [9]). The set of fundamental solutions for (4.16) is given by

$$
\left\{s^{-j+\frac{3-m}{2}}, s^{-j-\frac{3+m}{2}}\right\}, \quad j=0,1,2, \ldots
$$

Combining (4.15) and (4.16) we may compute the terms recursively by the formula

$$
B_{j+1}^{\prime}(s)=\frac{1}{2(j+1)} B_{j}(s), \quad j=0,1,2, \ldots
$$


Then, we have

$$
\begin{aligned}
& B_{1}(s)=\frac{1}{2 \times 1} \int B_{0}(s) d s \\
& B_{2}(s)=\frac{1}{2 \times 2} \times \frac{1}{2 \times 1} \iint B_{0}(s) d s d s \\
& B_{3}(s)=\frac{1}{2 \times 3} \times \frac{1}{2 \times 2} \times \frac{1}{2 \times 1} \iiint B_{0}(s) d s d s d s
\end{aligned}
$$

and in general

$$
B_{j}(s)=\frac{1}{2^{j} j !} \underbrace{\int \ldots \int B_{0}(s) d s \ldots d s}_{j \text { times }}, \quad j=0,1,2, \ldots,
$$

where

$$
B_{0}(s)=s^{\frac{3-m}{2}} \quad \text { or } \quad B_{0}(s)=s^{-\frac{3+m}{2}} .
$$

By induction it is possible to prove that

$$
\begin{aligned}
& \underbrace{\int \ldots \int B_{0}(s) d s \ldots d s}_{j \text { times }}=\frac{s^{\frac{3-m}{2}+j}}{\left(\frac{5-m}{2}\right)_{j}}, \quad \text { when } B_{0}(s)=s^{\frac{3-m}{2}}, \\
& \underbrace{\int \ldots \int B_{0}(s) d s \ldots d s}_{j \text { times }}=\frac{s^{-\frac{3+m}{2}+j}}{\left(-\frac{1+m}{2}\right)_{j}}, \quad \text { when } B_{0}(s)=s^{-\frac{3+m}{2}} .
\end{aligned}
$$

Therefore (4.19) takes the form

$$
\begin{aligned}
& B_{j}(s)=\frac{s^{\frac{3-m}{2}+j}}{2^{j} j !\left(\frac{5-m}{2}\right)_{j}}, \quad \text { when } B_{0}(s)=s^{\frac{3-m}{2}}, \\
& B_{j}(s)=\frac{s^{-\frac{3+m}{2}+j}}{2^{j} j !\left(-\frac{1+m}{2}\right)_{j}}, \quad \text { when } B_{0}(s)=s^{\frac{3-m}{2}},
\end{aligned}
$$

where in each case $m$ is chosen such that the argument of the pochhammer symbol is not a negative integer number. From (4.22) and (4.23) we get the following two possible expressions for $\Phi_{2}$

$$
\begin{aligned}
& \Phi_{2}(t, s)=\sum_{j=0}^{+\infty} \frac{t^{j} s^{\frac{3-m}{2}+j}}{2^{j} j !\left(\frac{5-m}{2}\right)_{j}}=s^{\frac{3-m}{2}}{ }_{0} F_{1}\left(\frac{5-m}{2}, \frac{s t}{2}\right), \\
& \Phi_{2}(t, s)=\sum_{j=0}^{+\infty} \frac{t^{j} s^{-\frac{3+m}{2}+j}}{2^{j} j !\left(-\frac{1+m}{2}\right)_{j}}=s^{-\frac{3+m}{2}}{ }_{0} F_{1}\left(-\frac{1+m}{2}, \frac{s t}{2}\right),
\end{aligned}
$$


when $B_{0}(s)=s^{\frac{3-m}{2}}$ or $B_{0}(s)=s^{\frac{3-m}{2}}$, respectively, and ${ }_{0} F_{1}(\alpha, z)$ is the confluent hypergeometric function defined by the following series (see [1])

$$
{ }_{0} F_{1}(\alpha, z)=\sum_{j=0}^{+\infty} \frac{z^{j}}{(\alpha)_{j} j !} .
$$

Moreover, in (4.24) and (4.25) the value of $m$ is chosen such that the first argument of ${ }_{0} F_{1}$ is not a negative integer number. From (4.24) and (4.25) we get, for each possible choice of $B_{0}$, the correspondent expressions for $v(a, b, c)$ (see $(4.5))$

$$
\begin{aligned}
& v(a, b, c)=\frac{b^{\frac{3-m}{2}}}{c}{ }_{0} F_{1}\left(\frac{5-m}{2}, \frac{a b}{2 c^{2}}\right), \\
& v(a, b, c)=\frac{c^{1+m}}{b^{\frac{3+m}{2}}}{ }_{0} F_{1}\left(-\frac{1+m}{2}, \frac{s t}{2}\right),
\end{aligned}
$$

where $t=\frac{a}{c}$ and $s=\frac{b}{c}$, and $m$ is in the conditions described previously. Now we find the expression for $A_{j}$. Taking into account (4.22) and (4.23), expression (4.12) takes the form

$$
\begin{aligned}
& A_{j}^{\prime}(s)=-\frac{(2 j+1) s^{\frac{3-m}{2}}+j}{2^{j+1} j !\left(\frac{5-m}{2}\right)_{j}}, \quad \text { when } B_{0}(s)=s^{\frac{3-m}{2}}, \\
& A_{j}^{\prime}(s)=-\frac{(j-1) s^{-\frac{3+m}{2}+j}}{2^{j} j !\left(-\frac{1+m}{2}\right)_{j}}, \quad \text { when } B_{0}(s)=s^{\frac{3-m}{2}}
\end{aligned}
$$

and therefore

$$
\begin{aligned}
& A_{j}(s)=-\frac{(2 j+1) s^{\frac{5-m}{2}+j}}{2^{j} j !(5-m+2 j)\left(\frac{5-m}{2}\right)_{j}}, \quad \text { when } B_{0}(s)=s^{\frac{3-m}{2}}, \\
& A_{j}(s)=\frac{(j-1) s^{-\frac{1+m}{2}+j}}{2^{j-1} j !(1+m-2 j)\left(-\frac{1+m}{2}\right)_{j}}, \quad \text { when } B_{0}(s)=s^{\frac{3-m}{2}},
\end{aligned}
$$

where in each case $m$ is chosen such that the argument of the pochhammer symbol is not a negative integer number. Now from (4.28) and (4.29) we can present the final expression for $\Phi_{1}$, when $B_{0}(s)=s^{\frac{3-m}{2}}$ or $B_{0}(s)=s^{\frac{3-m}{2}}$, respectively, and $m$ is even

$$
\begin{aligned}
\Phi_{1}(t, s) & =-\sum_{j=0}^{+\infty} \frac{t^{j}(2 j+1) s^{\frac{5-m}{2}+j}}{2^{j} j !(5-m+2 j)\left(\frac{5-m}{2}\right)_{j}} \\
& =-\frac{\Gamma\left(\frac{5-m}{2}\right)}{2}\left(s^{\frac{5-m}{2}}{ }_{0} \tilde{F}_{1}\left(\frac{7-m}{2}, \frac{s t}{2}\right)+t s^{\frac{7-m}{2}}{ }_{0} \tilde{F}_{1}\left(\frac{9-m}{2}, \frac{s t}{2}\right)\right),
\end{aligned}
$$


or

$$
\begin{aligned}
\Phi_{1}(t, s) & =\sum_{j=0}^{+\infty} \frac{t^{j}(j-1) s^{-\frac{1+m}{2}+j}}{2^{j-1} j !(1+m-2 j)\left(-\frac{1+m}{2}\right)_{j}} \\
& =\frac{\Gamma\left(-\frac{1+m}{2}\right)}{2}\left(2 s^{-\frac{1+m}{2}}{ }_{0} \tilde{F}_{1}\left(\frac{1-m}{2}, \frac{s t}{2}\right)-t s^{\frac{1-m}{2}}{ }_{0} \tilde{F}_{1}\left(\frac{3-m}{2}, \frac{s t}{2}\right)\right),
\end{aligned}
$$

where ${ }_{0} \tilde{F}_{1}(\alpha, z)$ is the regularized confluent hypergeometric function defined by the following series (see [1])

$$
{ }_{0} \tilde{F}_{1}(\alpha, z)=\sum_{j=0}^{+\infty} \frac{z^{j}}{\Gamma(\alpha+j) j !},
$$

and $m$ is chosen such that the first argument of ${ }_{0} \tilde{F}_{1}$ is not a negative integer number. From (4.30) and (4.31) we get, for each possible choice of $B_{0}$ and considering $m$ even, the correspondent expressions for $u(a, b, c)$ (see $(4.5)$ )

$$
\begin{aligned}
& u(a, b, c) \\
& =-\frac{\Gamma\left(\frac{5-m}{2}\right)}{2}\left(\frac{b^{\frac{5-m}{2}}}{c^{2}}{ }_{0} \tilde{F}_{1}\left(\frac{7-m}{2}, \frac{a b}{2 c^{2}}\right)+\frac{a b^{\frac{7-m}{2}}}{c^{4}}{ }_{0} \tilde{F}_{1}\left(\frac{9-m}{2}, \frac{a b}{2 c^{2}}\right)\right), \\
& u(a, b, c) \\
& =\frac{\Gamma\left(-\frac{1+m}{2}\right)}{2}\left(2 \frac{c}{b^{\frac{1+m}{2}}} \tilde{F}_{1}\left(\frac{1-m}{2}, \frac{a b}{2 c^{2}}\right)-\frac{a b^{\frac{1-m}{2}}}{c}{ }_{0} \tilde{F}_{1}\left(\frac{3-m}{2}, \frac{a b}{2 c^{2}}\right)\right),
\end{aligned}
$$

and $m$ is chosen such that the first argument of ${ }_{0} \tilde{F}_{1}$ is not a negative integer number. From the expressions obtained previously for the functions $u$ and $v$ (see (4.32), (4.33), (4.26) and (4.27)), we obtain the following two examples of axially symmetric $(\kappa, \tau)$-hypermonogenic functions, (which are only valid for the case when $m$ is even, and where $a=|\underline{x}|^{2}, b=|\underline{y}|^{2}$ and $\left.c=\langle\underline{x}, \underline{y}\rangle\right)$

$$
\begin{aligned}
& f(\mathbf{x}, \mathbf{y})=(\underline{x} A+\underline{y} B) e_{m} \\
& =\left(\underline{x} u\left(|\underline{x}|^{2},|\underline{y}|^{2},\langle\underline{x}, \underline{y}\rangle\right)+\underline{y} v\left(|\underline{x}|^{2},|\underline{y}|^{2},\langle\underline{x}, \underline{y}\rangle\right)\right) x_{m}^{\kappa} y_{m}^{\tau} e_{m} \\
& =\frac{|\underline{y}|^{3-m}}{\langle\underline{x}, \underline{y}\rangle}\left[-\frac{\Gamma\left(\frac{5-m}{2}\right)}{2} \underline{x}\left(\frac{|\underline{y}|^{2}}{\langle\underline{x}, \underline{y}\rangle^{2}}{ }_{0} \tilde{F}_{1}\left(\frac{7-m}{2}, \frac{|\underline{x}|^{2}|\underline{y}|^{2}}{2\langle\underline{x}, \underline{y}\rangle^{2}}\right)\right.\right. \\
& \left.\left.+\frac{|\underline{x}|^{2}|\underline{y}|^{4}}{\langle\underline{x}, \underline{y}\rangle^{3}}{ }_{0} \tilde{F}_{1}\left(\frac{9-m}{2}, \frac{|\underline{x}|^{2}|\underline{y}|^{2}}{2\langle\underline{x}, \underline{y}\rangle^{2}}\right)\right)+\underline{y}_{0} F_{1}\left(\frac{5-m}{2}, \frac{|\underline{x}|^{2}|\underline{y}|^{2}}{2\langle\underline{x}, \underline{y}\rangle^{2}}\right)\right] x_{m}^{\kappa} y_{m}^{\tau} e_{m},
\end{aligned}
$$


when $B_{0}(s)=s^{\frac{3-m}{2}}$, and

$$
\begin{aligned}
& f(\mathbf{x}, \mathbf{y})=(\underline{x} A+\underline{y} B) e_{m} \\
& =\left(\underline{x} u\left(|\underline{x}|^{2},|\underline{y}|^{2},\langle\underline{x}, \underline{y}\rangle\right)+\underline{y} v\left(|\underline{x}|^{2},|\underline{y}|^{2},\langle\underline{x}, \underline{y}\rangle\right)\right) x_{m}^{\kappa} y_{m}^{\tau} e_{m} \\
& =\left[\Gamma ( - \frac { 1 + m } { 2 } ) \underline { x } \left(\frac{\langle\underline{x}, \underline{y}\rangle}{|\underline{y}|^{1+m}}{ }_{0} \tilde{F}_{1}\left(\frac{1-m}{2}, \frac{|\underline{x}|^{2}|\underline{y}|^{2}}{2\langle\underline{x}, \underline{y}\rangle^{2}}\right)\right.\right. \\
& \left.-\frac{|\underline{x}|^{2}|\underline{y}|^{1-m}}{2\langle\underline{x}, \underline{y}\rangle}{ }_{0} \tilde{F}_{1}\left(\frac{3-m}{2}, \frac{|\underline{x}|^{2}|\underline{y}|^{2}}{2\langle\underline{x}, \underline{y}\rangle^{2}}\right)\right) \\
& \left.+\underline{y} \frac{\langle\underline{x}, \underline{y}\rangle^{1+m}}{|\underline{y}|^{3+m}}{ }_{0} F_{1}\left(-\frac{1+m}{2}, \frac{|\underline{x}|^{2}|\underline{y}|^{2}}{2\langle\underline{x}, \underline{y}\rangle^{2}}\right)\right] x_{m}^{\kappa} y_{m}^{\tau} e_{m},
\end{aligned}
$$

when $B_{0}(s)=s^{\frac{3-m}{2}}$. In both expressions and $m$ is chosen such that the first argument of the hypergeometric functions ${ }_{0} \tilde{F}_{1}$ and ${ }_{0} F_{1}$ is not a negative integer number.

\section{Conclusions}

In this paper we started to study $(\kappa, \tau)$-hypermonogenic functions, which are hypermonogenic generalizations of monogenic functions of two vector variables defined in [11]. We also started to look for axially symmetric solutions for the system obtaining some simple examples. These non-trivial solutions guarantee that the theory is well defined and worth to study further. The complete study of axially symmetric functions we leave to the future. A more general class of axially symmetric solutions is obtained multiplying our axially symmetric functions by a $(k, l)$-homogeneous polynomial $P_{k, l}(\underline{x}, \underline{y})$ (see $[11,12])$. This will be also an interesting topic in future.

\section{Acknowledgment}

The third author was supported by Portuguese funds through the CIDMA - Center for Research and Development in Mathematics and Applications, and the Portuguese Foundation for Science and Technology ("FCT-Fundação para a Ciência e a Tecnologia"), within project UID/MAT/ 0416/2013.

N. Vieira is Auxiliar Researcher, under the FCT Researcher Program 2014 (Ref: IF/00271/2014).

The second author would like to thank all the colleagues in Campus de Santiago for the excellent coffee, the patience, the hospitality and the inspirational atmosphere. 


\title{
References
}

[1] M. Abramowitz and I.A. Stegun, Handbook of mathematical functions with formulas, graphs, and mathematical tables. National Bureau of Standards, WileyInterscience Publication, John Wiley \& Sons, New York etc., 1972.

[2] F. Brackx, R. Delanghe and F. Sommen, Clifford analysis. Research Notes in Mathematics-Vol.76, Pitman Advanced Publishing Program, Boston - London - Melbourne, 1982.

[3] R. Delanghe, F. Sommen and V. Souček, Clifford algebra and spinor-valued functions. A function theory for the Dirac operator. Related REDUCE software by F. Brackx and D. Constales. Mathematics and its Applications-Vol.53, Kluwer Academic Publishers, Dordrecht etc, 1992.

[4] S.L. Eriksson and H. Leutwiler, Hypermonogenic functions in Clifford analysis and their applications in mathematical physics. Clifford Anal. 2 (2000), 287-302.

[5] H. Leutwiler, Generalized Function Theory. Clifford Analysis and Applications (Tampere 2006), Tampere Univ. of Tech., Inst. of Math., Research Report No.82 (2006), 1-23.

[6] H. Leutwiler, Modified Clifford analysis. Complex Variables, Theory Appl. 17No.3-4 (1992), 153-171.

[7] H. Leutwiler, Modified quaternionic analysis in $\mathbb{R}^{3}$. Complex Variables 20-No.14 (1992), 19-51.

[8] H. Orelma, Harmonic forms on conformal Euclidean manifolds: the Clifford multivector approach. Adv. Appl. Clifford Algebr. 22-No.1 (2012), 143-158.

[9] A.D. Polyanin and V.F. Zaitsev, Handbook of exact solutions for ordinary differential equations. 2nd. ed.. CRC Press, Boca Raton, FL, 2003.

[10] A.D. Polyanin, V.F. Zaitsev and A. Moussiaux, Handbook of first order partial differential equations. Differential and Integral Equations and Their Applications-Vol.1.. Taylor \& Francis, London, 2002.

[11] F. Sommen, Clifford analysis in two and several vector variables. Appl. Anal. 73-No.1-2 (1999), 225-253.

[12] F. Sommen and N. Van Acker, Functions of two vector variables. Adv. Appl. Clifford Algebr. 4-No.1 (1994), 65-72.

[13] F. Sommen and N. Van Acker, Monogenic differential operators. Results Math. 22-No.3-4 (1992), 781-798.

[14] L. Van de Voorde, Higher spin dirac operators in two vector variables in Clifford analysis. PhD Thesis, University of Gent, Belgium, 2011.

\author{
S.-L. Eriksson \\ Department of Mathematics and Statistics \\ University of Helsinki \\ P.O. Box 68, FI-00014 Helsinki, Finland. \\ e-mail: sirkka-liisa.eriksson@helsinki.fi
}




\section{H. Orelma}

Laboratory of Civil Engineering

Faculty of Business and Built Environment

Tampere University of Technology

Korkeakoulunkatu 10, 33720 Tampere, Finland.

e-mail: heikki.orelma@tut.fi

\section{N. Vieira}

CIDMA - Center for Research and Development in Mathematics and Applications Department of Mathematics, University of Aveiro

Campus Universitário de Santiago, 3810-193 Aveiro, Portugal.

e-mail: nloureirovieira@gmail.com 